\title{
A giant splenic artery aneurysm
}

\author{
Georgios Varnavas MD, Christodoulos Dolapsakis MD
}

Cite as: CMAJ 2020 June 1;192:E608. doi: 10.1503/cmaj.191180

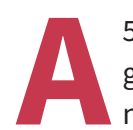

51-year-old postmenopausal woman presented to the emergency department with a 2-day history of melena. She had no history of alcohol abuse and was not taking any medications. Physical examination found a nontender pulsative epigastric mass, and a digital rectal examination found melena. An upper endoscopy showed narrowing of gastric body lumen but was otherwise normal. Ultrasonography of her abdomen (Figure 1) showed a well-defined epigastric mass adjacent to the spleen, suggestive of an aneurysm. Contrast-enhanced computed tomography (Figure 2) confirmed the presence of an aneurysm $\left(7.7 \times 10 \times 6 \mathrm{~cm}^{3}\right.$ in diameter) of the splenic artery with a mural thrombus $(5.2 \mathrm{~cm}$ in size), adherent to the stomach and pancreas, without signs of portal hypertension.

The patient underwent aneurysmectomy with splenectomy, distal pancreatectomy and sleeve gastrectomy because of the erosion of the stomach wall from the aneurysm. Histopathology confirmed a true aneurysm of the splenic artery. Her postoperative course was uneventful, and we discharged the patient to home on the seventh day of admission.

True aneurysm of the splenic artery (i.e., containing all 3 layers of the vessel wall) is the most common visceral vessel aneurysm. Although aneurysms of the great vessels are more frequent in men, aneurysms of the splenic artery are more common in women: onequarter of them occur in pregnancy, and most are diagnosed at the time of rupture. ${ }^{1}$ Hormonal changes and increased arteriovenous shunting in the splenic vessels during pregnancy may explain this predisposition. ${ }^{2}$ Other risk factors include portal hypertension and liver transplantation. Atherosclerosis is a common finding, but no causal relation has been established. ${ }^{3}$

Aneurysms of the splenic artery may rupture freely into the peritoneum or erode adjacent structures (e.g., stomach, pancreatic duct, splenic vein and colon) and present as gastrointestinal bleeding as in our patient. Symptomatic aneurysms and those with highrisk characteristics for rupture (e.g., lesion $>2 \mathrm{~cm}$ in size, pregnancy or portal hypertension) should be treated. Open surgery is the traditional treatment; however, endovascular management has become the preferred choice where available. ${ }^{4}$

\section{References}

1. Bloom SL, Uppot R, Roberts DJ. Case records of the Massachusetts General Hospital. Case 32-2010. A pregnant woman with abdominal pain and fluid in the peritoneal cavity. N Engl J Med 2010;363:1657-65.

2. Abbas MA, Stone WM, Fowl RJ, et al. Splenic artery aneurysms: two decades experience at Mayo Clinic. Ann Vasc Surg 2002;16:442-9.

3. Rao S, Sivina M, Willis I, et al. Massive lower gastrointestinal tract bleeding due to splenic artery aneurysm: a case report. Ann Vasc Surg 2007;21:388-91.

4. Li ES, Mu JX, Ji SM, et al. Total splenic artery embolization for splenic artery aneurysms in patients with normal spleen. World J Gastroenterol 2014;20:555-60.

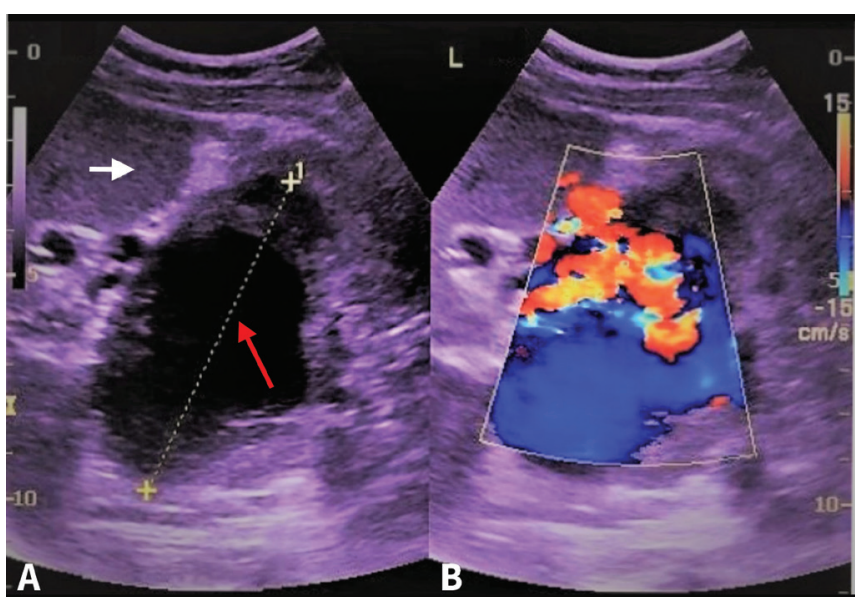

Figure 1: (A) Ultrasonography showing a well-defined mass (red arrow) adjacent to the spleen (white arrow) in a 51-year-old woman with melena. (B) Colour Doppler ultrasonography showing turbulent blood flow suggestive of an aneurysm.

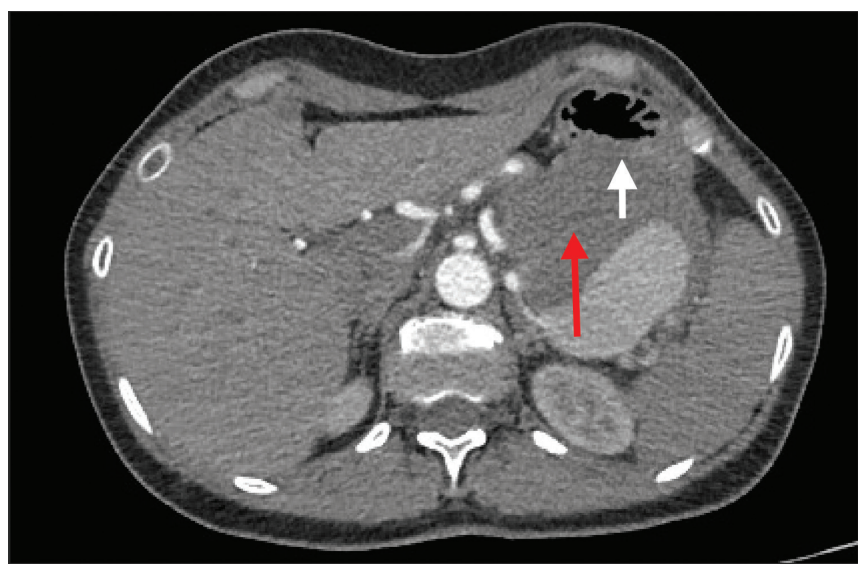

Figure 2: Contrast-enhanced computed tomography showing a splenic artery aneurysm, with a mural thrombus (red arrow) causing anterior displacement of the stomach (white arrow).

\section{Competing interests: None declared.}

This article has been peer reviewed.

The authors have obtained patient consent.

Affiliation: Internal Medicine, Evaggelismos General Hospital, Athens, Greece

Correspondence to: Christodoulos Dolapsakis, dolapsak@yahoo.gr 